\title{
Dynamic Evolution Analysis of Stock Price Fluctuation and Its Control
}

\author{
Yuhua Xu $\mathbb{D}^{1},{ }^{1}$ Zhongyi Ke, ${ }^{1}$ Chengrong Xie $\mathbb{D},{ }^{2}$ and Wuneng Zhou $\mathbb{D I}^{3}$ \\ ${ }^{1}$ School of Finance, Nanjing Audit University, Jiangsu 211815, China \\ ${ }^{2}$ School of Science, Nanjing Audit University, Jiangsu 211815, China \\ ${ }^{3}$ College of Information Science and Technology, Donghua University, Shanghai 201620, China \\ Correspondence should be addressed to Chengrong Xie; xcr0603@163.com
}

Received 24 August 2017; Revised 3 November 2017; Accepted 15 November 2017; Published 29 January 2018

Academic Editor: Danilo Comminiello

Copyright (c) 2018 Yuhua Xu et al. This is an open access article distributed under the Creative Commons Attribution License, which permits unrestricted use, distribution, and reproduction in any medium, provided the original work is properly cited.

This paper studies a simple dynamical system of stock price fluctuation time series based on the rule of stock market. When the stock price fluctuation system is disturbed by external excitations, the system exhibits obviously chaotic phenomena, and its basic dynamic properties are analyzed. At the same time, a new fixed-time convergence theorem is proposed for achieving fixed-time control of stock price fluctuation system. Finally, the effectiveness of the method is verified by numerical simulation.

\section{Introduction}

Since Lorenz found the first chaotic attractor [1], a variety of chaotic systems have been found, and in particular chaotic phenomenon also appeared in the economic system; for example, Chen discovered the existence of chaotic attractor in stock market [2]. Xie et al. studied chaos synchronization of financial chaotic system with external perturbation [3]. $\mathrm{Ma}$ et al. studied chaotic financial Cournot model with dual-channel recycling and uncertain consumer perception [4]. Despite the existence of chaotic phenomena in the economic system, but the development of dynamic model in the economy is relatively slow; the main reason is that there are some difficulties to establish complex dynamic system by abstracting the economic phenomenon. Recently, some researchers discussed stock price problem by dynamic method; for example, Li et al. considered the problem of the dynamic pricing and the periodic ordering for deteriorating items with a stochastic inventory level depending on the stock-dependent demand and the selling price [5]. Grassetti and Hunanyan discussed the economic growth theory with Kadiyala production function [6]. Arévalo et al. discussed the dynamic trading rule based on filtered flag pattern recognition for stock market price forecasting [7]. Zhang et al. studied dynamic static pricing in a supply chain with advertising [8]. Primbs and Barmish presented robustness of simultaneous long-short stock trading control with timevarying price dynamics [9]. In [5-9], although the authors studied the price index problem with dynamics, it did not discuss the price fluctuation problem from the nonlinear point of view; in particular the external excitations problem of the price fluctuation system was not discussed. However, the nonlinear phenomenon of price fluctuation and the external excitations of the dynamic system [10] are common in practice. Therefore, it is meaningful to discuss the nonlinear characteristics of price fluctuation system under external excitations.

In addition, the chaos is sometimes harmful to the dynamical system in some cases, so it is essential for the effective control of the dynamical system. The various methods of chaos control have been put forward by researchers [11-16]; these control methods were mostly based on infinite time control. In fact, complex systems are usually required to be controlled in a certain amount of time, the finite time control became an important index to measure the quality of the control criteria, and it not only has important theoretical significance and also has important practical value [17]. However, the stable time of finite time control depends largely on the initial state of the system, so there are some limitations in the practical application. In order to compensate for the 
finite time control, Polyakov proposed the fixed-time stability of the system [18], and the stability is as follows: (1) fixed time guaranteed that the system is the finite time stability without regard to initial conditions. Hence, the study of fixed-time control has a wide range of research interests, and more and more fixed-time control methods were also proposed [1922]. In particular, in [23], Zuo proposed a class of fixed-time stability theory for the differential equation: $\dot{y}=-\alpha y^{2-p / q}-$ $\beta y^{p / q}$, which was fixed-time stable at $T \leq q \pi / 2 \sqrt{\alpha \beta}(q-p)$. Subsequently, the theorem proposed by Zuo was used to discuss the fixed-time control of dynamical systems; please see [21, 24-27]. This paper attempts to propose a new finite time convergence theory to discuss the control problem of stock price fluctuation system, the new fixed-time stability method proposed in this paper converged faster than the proposed method by Zuo, and the new fixed-time stable theorem contains the theorem proposed by Zuo, so the new fixed-time stable theorem can be applied more widely.

Motivated by the existing works, the aim of this paper is to discuss dynamic evolution of stock price fluctuation and its control. The main contributions of this paper are as follows: (1) a stock price fluctuation dynamical system is established under the influence of external excitations, and its basic dynamic behavior is analyzed. (2) A new fixedtime convergence theorem is proposed. (3) According to the new fixed-time convergence theorem, the fixed-time control criterion of the stock price fluctuation dynamical system is given.

\section{Model and Preliminaries}

In this paper, $A_{b}(t)$ denotes stock purchases at time $t$, namely, the purchase amount, $A_{s}(t)$ denotes stock selling amount, $P(t)$ denotes the stock price at time $t$, let $P(t)=p_{0}+p(t), p_{0}$ denotes the original stock price, $p(t)$ denotes the stock price fluctuation value, $p(t)>0$ means prices rose, $p(t)<0$ means prices down, and $p(0)=0 . b_{i}(i=1,2,3,4)$ and $s_{i}(i=1,2)$ denote the undetermined coefficient.

According to the literature [28], the difference between supply and demand of the stock at time $t$ is described in mathematical model as follows:

$$
A(t)=A_{b}(t)-A_{s}(t) .
$$

Assume that all variables are continuous functions about time $t$, according to (1):

$$
A(t) \rightleftharpoons P(t+1)-P(t)=\dot{P}(t) .
$$

As long as the introduction coefficient meets dimensional requirements, formula (2) can be written into an equation; that is,

$$
\dot{P}(t)=k A(t) \text {. }
$$

Assuming a risk neutral, "buy low sell high" strategy is to be executed in the stock market. According to the law of the stock market, $A_{b}(t)$ is inversely proportional to $P(t)$, and $A_{b}(t)$ is proportional to the negative value of $\dot{P}(t)$. Thus the equation is obtained:

$$
\dot{A}_{b}(t)=b_{1}(P(t))^{-1}-b_{2} \dot{P}(t) .
$$

In $(4)$, if $\dot{P}(t) \approx P(t+1)-P(t)>0$, that is, $P(t+1)>$ $P(t)$, then the amount of stock purchase should be reduced; on the other hand, the amount of stock purchased should be increased for $\dot{P}(t) \approx P(t+1)-P(t)<0$. However, the rate of change in stock buying not only is related to the price of stock and the rate of stock price change at the moment $t$ but also has a direct relationship with the buying and selling volume of stock at the moment $t$. Let the rate of change in stock buying be proportional to the buying amount at the moment $t$ and be proportional to the negative value of the stock sold. Thus the equation is obtained:

$$
\begin{aligned}
\dot{A}_{b}(t)= & b_{1}(P(t))^{-1}-b_{2} \dot{P}(t)+b_{3} A_{b}(t)-b_{4} A_{s}(t) \\
& +\delta_{1},
\end{aligned}
$$

where $\delta_{1}$ denotes the outside market interference, such as the impact of external policy.

Similar to (5), the rate of change for selling volume $A_{s}(t)$ is directly proportional to the stock price and is proportional to the rate of change for the stock price; meanwhile, $A_{s}(t)$ is proportional to the selling amount at time $t$, and it is proportional to the negative value of the stock buying. Thus the equation is obtained:

$$
\dot{A}_{s}(t)=s_{1} P(t)+s_{2} \dot{P}(t)+b_{3} A_{s}(t)-b_{4} A_{b}(t)+\delta_{2},
$$

similar to $\delta_{1}, \delta_{2}$ denotes the outside market interference.

Let $\dot{P}(t)=k\left(A_{b}(t)-A_{s}(t)\right)$ and $\ddot{P}(t)=k\left(\dot{A}_{b}(t)-\dot{A}_{s}(t)\right)$.

From (1)-(6), we have

$$
\begin{aligned}
& \ddot{P}(t)=k\left(b_{1}(P(t))^{-1}-b_{2} \dot{P}(t)+b_{3} A_{b}(t)-b_{4} A_{s}(t)\right. \\
& \left.+\delta_{1}-s_{1} P(t)-s_{2} \dot{P}(t)-b_{3} A_{s}(t)+b_{4} A_{b}(t)-\delta_{2}\right) \\
& =k b_{1}(P(t))^{-1}-k\left(b_{2}+s_{2}\right) \dot{P}(t)+\left(k b_{3}+k b_{4}\right) \\
& \cdot\left(A_{b}(t)-A_{s}(t)\right)-k s_{1} P(t)+k\left(\delta_{1}-\delta_{2}\right) \\
& =-k s_{1} P(t)+k b_{1}(P(t))^{-1}+\left[\left(b_{3}+b_{4}\right)\right. \\
& \left.-k\left(b_{2}+s_{2}\right)\right] \dot{P}(t)+k\left(\delta_{1}-\delta_{2}\right) .
\end{aligned}
$$

Therefore, the dynamic equation of stock price fluctuation $p(t)$ is

$$
\begin{aligned}
\ddot{p}(t)= & -k s_{1}\left(p_{0}+p(t)\right)+k b_{1}\left(p_{0}+p(t)\right)^{-1} \\
& +\left[\left(b_{3}+b_{4}\right)-k\left(b_{2}+s_{2}\right)\right] \dot{p}(t)+k\left(\delta_{1}-\delta_{2}\right) \\
= & -k s_{1} p(t)+k b_{1}\left(p_{0}+p(t)\right)^{-1} \\
& +\left[\left(b_{3}+b_{4}\right)-k\left(b_{2}+s_{2}\right)\right] \dot{p}(t)+k\left(\delta_{1}-\delta_{2}\right) \\
& -k s_{1} p_{0} .
\end{aligned}
$$

Let $a_{1}=k s_{1}, a_{2}=k b_{1}, a_{3}=\left(b_{3}+b_{4}\right)-k\left(b_{2}+s_{2}\right)$, and $k\left(\delta_{1}-\delta_{2}-s_{1} p_{0}\right)=q \cos w t$, and then the dynamic equation of stock price fluctuation $p(t)$ can be reduced to

$$
\begin{aligned}
\ddot{p}(t)= & -a_{1} p(t)+a_{2}\left(p_{0}+p(t)\right)^{-1}+a_{3} \dot{p}(t) \\
& +q \cos w t,
\end{aligned}
$$


where $p(t)$ represents the state of stock price fluctuation, $\ddot{p}(t)$ represents the acceleration of stock price fluctuation, $q$ represents the control parameter of the policy, and $w$ represents the frequency of external excitations.

To facilitate the analysis of model (9), it can be described in the following:

$$
\begin{aligned}
\dot{x}(t)= & y(t) \\
\dot{y}(t)= & -a_{1} x(t)+a_{2}\left(p_{0}+x(t)\right)^{-1}+a_{3} y(t) \\
& +q \cos w t,
\end{aligned}
$$

where $p(t)=x(t)$.

In the practical application, $p_{0}+x(t)>0$, the stock price fluctuation cannot make $p_{0}+x(t) \leq 0$; otherwise, it will go bankrupt or withdrawn from the market, but it is possible for the $p_{0}+x(t)<0$ in mathematical expression (10); when $p_{0}+$ $x(t)=0,\left(p_{0}+x(t)\right)^{-1}$ can be meaningless. In order to facilitate the study of theory and simulation, let $p_{0}=x(t) \operatorname{sign} x(t)+$ $a_{0}-x(t), a_{0}>0$, and (10) become the following equations:

$$
\begin{aligned}
\dot{x}(t)= & y(t) \\
\dot{y}(t)= & -a_{1} x(t)+a_{2}\left(a_{0}+x(t) \operatorname{sign} x(t)\right)^{-1}+a_{3} y(t) \\
& +q \cos w t .
\end{aligned}
$$

Remark 1. Although this assumption about (11) is flawed, (11) have the same properties as (10) for $x(t)>0$. Therefore, we can deduce the part evolutionary behavior of the stock price fluctuation $p(t)$ in practice by analyzing (11). Thus it can provide some theoretical reference for managers.

Remark 2. The dynamic method are often utilized to depict the complicated systems in the real world [29], and the nonlinear phenomenon of price fluctuation and the external excitations of the system [10] are common in practice. Therefore, it is meaningful to discuss the nonlinear characteristics of price fluctuation dynamical system under external excitations by dynamic method.

\section{Some Basic Properties of the Dynamics Model}

In this section, we will investigate some basic properties of (11).

3.1. Equilibria. Obviously, system (11) is a nonlinear autonomous system:

$$
\begin{aligned}
& y(t)=0 \\
& -a_{1} x(t)+a_{2}\left(a_{0}+x(t) \operatorname{sign} x(t)\right)^{-1}+a_{3} y(t) \\
& \quad+q \cos w t=0 .
\end{aligned}
$$

(1) When $x(t)=0$ and $a_{2}\left(a_{0}\right)^{-1}+q \cos w t=0$, the equilibrium point is $(x, y)=(0,0)$.

(2) When $x(t) \neq 0$ and $q \cos w t \neq 0$, the equilibrium points are

$$
(x, y)=\left(\frac{-\left(a_{0} a_{1}-q \operatorname{sign} x \cos w t\right) \pm \sqrt{\left(a_{0} a_{1}-q \operatorname{sign} x \cos w t\right)^{2}+4 a_{1} \operatorname{sign} x\left(a_{2}+a_{0} q \cos w t\right)}}{2 a_{1} \operatorname{sign} x}, 0\right) .
$$

(3) When $x(t) \neq 0$ and $q \cos w t=0$, the equilibrium points are

$$
(x, y)=\left(\frac{-a_{0} a_{1} \pm \sqrt{\left(a_{0} a_{1}\right)^{2}+4 a_{1} a_{2} \operatorname{sign} x}}{2 a_{1} \operatorname{sign} x}, 0\right) .
$$

Equations (11) are linearized, and the Jacobian matrix is defined as

$$
J=\left(\begin{array}{cc}
0 & 1 \\
-a_{1}-\frac{a_{2} \operatorname{sign} x}{\left(a_{0}+x \operatorname{sign} x\right)^{2}} & a_{3}
\end{array}\right) .
$$

To gain its eigenvalues, we let $|\lambda E-J|=0$, so the corresponding eigenvalues are

$$
=\frac{a_{3} \pm \sqrt{a_{3}^{2}-4\left(a_{1}+a_{2} \operatorname{sign} x /\left(a_{0}+x \operatorname{sign} x\right)^{2}\right)}}{2} .
$$

According to (16), the eigenvalues are only related to the abscissa of the equilibrium points, so there are the following conclusions:

(i) When $a_{3}>0$, all the equilibrium points are unstable.

(ii) As $\left(a_{0}+x \operatorname{sign} x\right)^{2}>0$, as long as you get suitable $a_{1}, a_{2}$ make $a_{1}+a_{2} \operatorname{sign} x /\left(a_{0}+x \operatorname{sign} x\right)^{2}<0$, and all the equilibrium points are unstable.

(iii) All the equilibrium points are stable except for two cases (i)-(ii).

3.2. Dissipativity and the Existence of Attractor. For system (11), we can obtain

$$
\nabla V=\frac{\partial \dot{x}}{\partial x}+\frac{\partial \dot{y}}{\partial y}=a_{3},
$$

when $a_{3}$ is a negative value, system (11) is a dissipative system, and an exponential contraction of system (11) is

$$
\frac{d V}{d t}=e^{a_{3}}
$$


In system (11), a volume element $V_{0}$ is apparently contracted by the flow into a volume element $V_{0} e^{a_{3} t}$ at time $t$. It means that each volume containing the trajectory of the systems shrinks to zero as $t \rightarrow \infty$ at an exponential rate $a_{3}$. So, all the systems orbits are eventually confined to a specific subset that have zero volume; the asymptotic motion settles onto an attractor of system (11) [30].

3.3. Lyapunov Exponent. Any system containing at least one positive Lyapunov exponent is defined to be chaotic [30]. The Lyapunov exponent spectrum of system (11) is found to be $L_{1}=3.1452$ and $L_{2}=3.4912$ for initial value $(0.01,0.1)$ and $a_{0}=0.15, a_{1}=0.6, a_{2}=0.5, a_{3}=-0.2, q=0.8$, and $w=0.6$. In addition, the Lyapunov dimension of the system is

$$
\begin{aligned}
D_{L} & =j+\frac{1}{\left|L_{j+1}\right|} \sum_{i=1}^{j} L_{i}=1+\frac{L_{1}}{\left|L_{2}\right|}=1+\frac{3.1452}{|3.4912|} \\
& =1.9009
\end{aligned}
$$

which means that system (11) is really a dissipative system and the Lyapunov dimension of the system is fractional.

\subsection{Poincare Map, Spectrum Map, and Bifurcation Diagram.} By simulation, system (11) exhibits some complex dynamical properties; for example, let $a_{0}=0.15, a_{1}=0.6, a_{2}=$ $0.5, a_{3}=-0.2, q=0.8$, and $w=0$, the stock market is subject to constant external excitations interference, and the market is in stable state (see Figure 1). If the external frequency parameter $w \neq 0$, that is, the market is disturbed by the changing external excitations, then system (11) gradually loses stability, produces bifurcations, and eventually produces chaos (see Figure 2; $w=0.6$ ).

When the parameters change, the bifurcation diagram would be far better to summarize all of the possible behaviors. For $q \in[0,1]$, the bifurcation diagram of system (11) shows the complicated bifurcation phenomena in Figure 3. The maximum Lyapunov exponent diagram is shown in Figure 4. Figure 4 shows that the system has some periodic windows, which play a very important role in the dynamics evolution of the system. In addition, Figure 5 shows the continuous broadband features of the system. Figure 6 shows the Poincare diagram of the system; it is clear that some sheets are folded, which also leads to complex dynamical behavior of the system.

Remark 3. In [5-9], although the authors analyzed some dynamical properties of price indices based on dynamics, some nonlinear properties of the price fluctuation system were not considered, such as the equilibrium point and stability, Lyapunov exponent, fractal dimension, and bifurcation diagram.

\section{Fixed-Time Control of the Dynamics Model}

In this section, we focus on studying fixed-time control of the stock price fluctuation model. To obtain the main results, we need the following preliminaries.

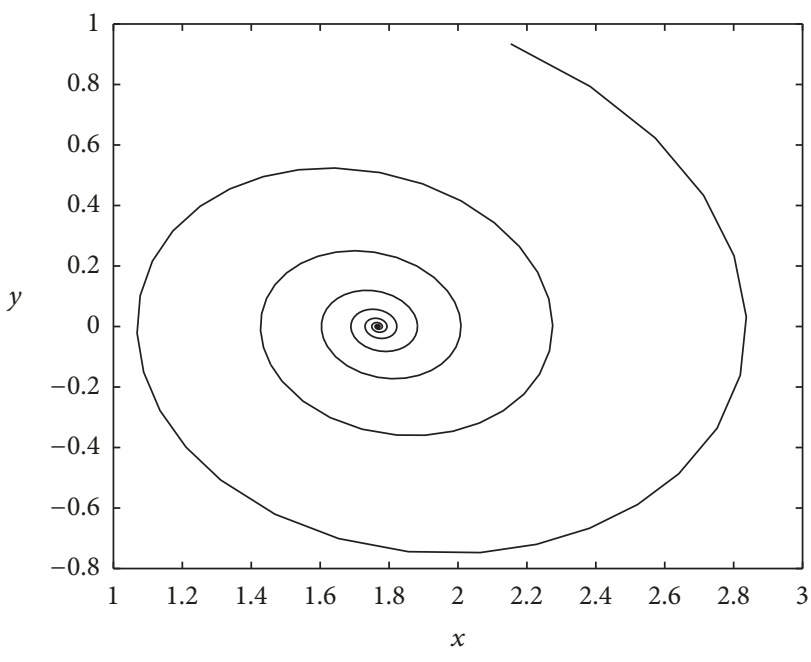

FIgURE 1: Phase diagram for $w=0$.

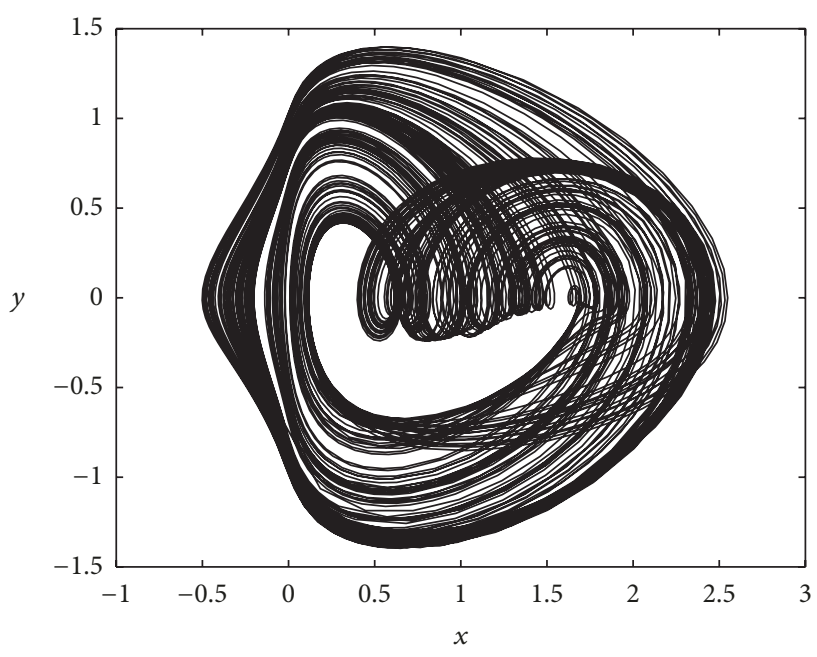

Figure 2: Phase diagram for $w=0.6$.

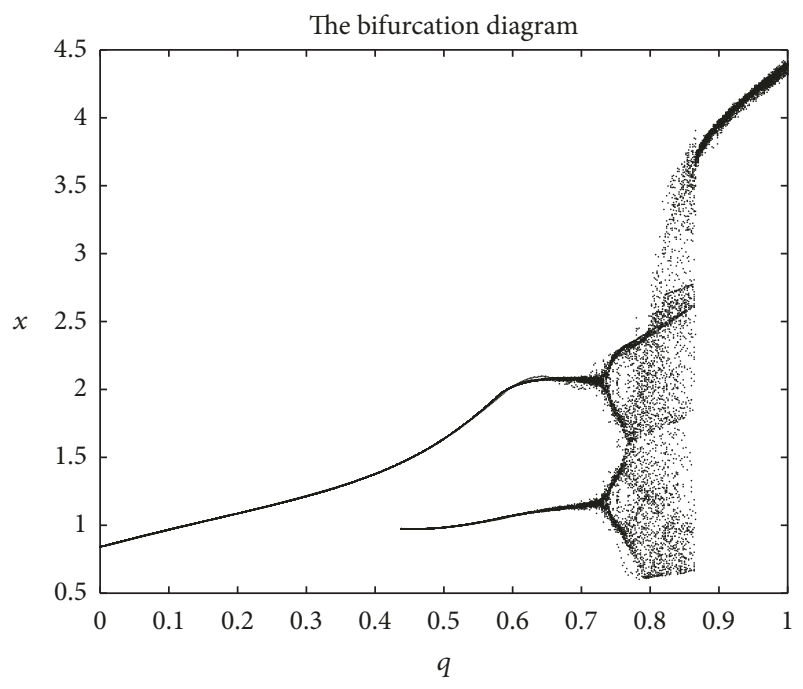

Figure 3: Bifurcation diagram. 


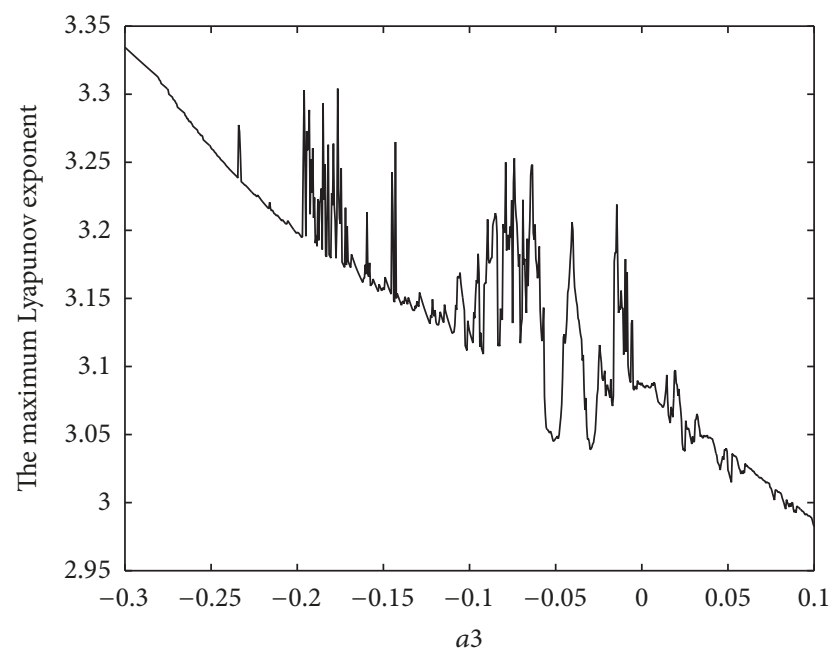

FIGURE 4: The maximum Lyapunov exponent diagram.

Lemma 4 (see [31]). For $x_{i} \in R, i=1,2, \ldots, n, 0<p \leq 1$, and then

$$
\left(\sum_{i=1}^{n}\left|x_{i}\right|\right)^{p} \leq \sum_{i=1}^{n}\left|x_{i}\right|^{p} \leq n^{1-p}\left(\sum_{i=1}^{n}\left|x_{i}\right|\right)^{p} .
$$

Lemma 5 (see [23]). Consider the following differential equation:

$$
\dot{y}=-\alpha y^{2-p / q}-\beta y^{p / q}
$$

where $\alpha>0, \beta>0$, and $p, q$ are positive odd integers, $p<q$. Then, system (21) is fixed-time stable, and

$$
T_{1} \leq \frac{q \pi}{2 \sqrt{\alpha \beta}(q-p)} .
$$

Based on Lemma 5, we obtain Lemma 6.

Lemma 6. Consider the following differential equation:

$$
\dot{y}=-\alpha y^{\delta-p / q}-\beta y^{p / q}
$$

where $\alpha>\beta>0, \delta>(p+q) / q, p<q$, and $p, q$ are coprime positive integers. Then, system (23) is fixed-time stable, and

$$
T<\frac{1}{\alpha} \frac{1}{\delta-(p+q) / q}+\frac{1}{\beta} \frac{q}{q-p} .
$$

Proof. Let $z=y^{1-p / q}$, then $(q /(q-p)) \dot{z} y^{p / q}=\dot{y}$, and system (23) can be rewritten as

$$
\dot{z}+\frac{q-p}{q} \alpha z^{q /(q-p) \cdot(\delta-2 p / q)}+\frac{q-p}{q} \beta=0 .
$$

That is,

$$
\frac{1}{\beta+\alpha z^{q /(q-p) \cdot(\delta-2 p / q)}} d z=-\frac{q-p}{q} d t .
$$

Solving (26) for convergence time, the upper bound of convergence time can be estimated as

$$
\begin{aligned}
& \lim _{z_{0} \rightarrow \infty} T\left(z_{0}\right) \\
& \quad=\lim _{z_{0} \rightarrow \infty} \frac{q}{q-p}\left(\int_{1}^{z_{0}} \frac{1}{\alpha z^{q /(q-p) \cdot(\delta-2 p / q)}+\beta} d z\right. \\
& \left.\quad+\int_{0}^{1} \frac{1}{\alpha z^{q /(q-p) \cdot(\delta-2 p / q)}+\beta} d z\right) .
\end{aligned}
$$

As $z \in[0,1]$, then $\beta<\alpha z^{q /(q-p) \cdot(\delta-2 p / q)}+\beta$.

So,

$$
\begin{aligned}
& \lim _{z_{0} \rightarrow \infty} T\left(z_{0}\right) \\
& \quad \leq \lim _{z_{0} \rightarrow \infty} \frac{q}{q-p}\left(\int_{1}^{z_{0}} \frac{1}{\alpha z^{q /(q-p) \bullet(\delta-2 p / q)}+\beta} d z\right. \\
& \left.\quad+\int_{0}^{1} \frac{1}{\beta} d z\right)<\lim _{z_{0} \rightarrow \infty} \frac{q}{q-p} \int_{1}^{z_{0}} \frac{1}{\alpha z^{q /(q-p) \cdot(\delta-2 p / q)}} d z \\
& +\frac{q}{(q-p)} \frac{1}{\beta} .
\end{aligned}
$$

When $z>1$ and $\delta>(p+q) / q$, $\lim _{z_{0} \rightarrow \infty} \int_{1}^{z_{0}}\left(1 / z^{q /(q-p) \cdot(\delta-2 p / q)}\right) d z<1 /(q /(q-p) \cdot(\delta-$ $2 p(q)-1)$, and we have

$$
\begin{aligned}
\lim _{z_{0} \rightarrow \infty} T\left(z_{0}\right)< & \frac{q}{q-p} \frac{1}{\alpha(q /(q-p) \cdot(\delta-2 p / q)-1)} \\
& +\frac{q}{q-p} \frac{1}{\beta} \\
= & \frac{1}{\alpha} \frac{1}{\delta-(p+q) / q}+\frac{1}{\beta} \frac{q}{q-p} .
\end{aligned}
$$

The proof is completed.

Remark 7. When $\delta=2$, Lemma 6 is reduced to Lemma 5 .

Remark 8. If $\delta>(p+q) / q, \beta<\alpha<\left(\pi^{2} / 4\right) \beta$, and then

$$
\begin{aligned}
T & =\frac{1}{\alpha} \frac{1}{\delta-(p+q) / q}+\frac{q}{q-p} \frac{1}{\beta} \\
& <\frac{1}{\alpha} \frac{q}{p-q}+\frac{q}{(q-p)} \frac{1}{\beta}=\frac{q}{q-p}\left(\frac{1}{\beta}-\frac{1}{\alpha}\right) \\
& <\frac{q}{q-p} \cdot \frac{1}{\beta}<\frac{q \pi}{2 \sqrt{\alpha \beta}(q-p)}
\end{aligned}
$$

that is, the fixed-time stable of the system presented in this paper achieves more faster convergence than Lemma 5.

Remark 9. Some researchers have discussed the fixed-time control problem of dynamical systems by using the theorem proposed by Zuo [21, 24-27]. From Remark 8, the fixedtime stable theorem of the dynamical system proposed in this paper achieves more faster convergence than the theorem proposed by Zuo and in [21, 24-27]. 
Remark 10. In Lemma 6 , as $\delta$ can take any value greater than $2 p / q$, and $2 p / q<2$, Lemma 6 contains Lemma 5 proposed by Zuo, and Lemma 5 can be applied more widely.

Based on Lemma 6, we analyze the fixed-time control problem of system (11).

If system (11) is the driving system, the response system is

$$
\begin{aligned}
\dot{x}_{1}(t)= & y_{1}(t)+u_{1}, \\
\dot{y}_{1}(t)= & -a_{1} x_{1}(t)+a_{2}\left(a_{0}+x_{1}(t) \operatorname{sign} x_{1}(t)\right)^{-1} \\
& +a_{3} y_{1}(t)+q \cos w t+u_{2} .
\end{aligned}
$$

According to system (11) and (31), the error system is

$$
\begin{aligned}
\dot{e}_{1}(t)= & e_{2}(t)+u_{1}, \\
\dot{e}_{2}(t)= & -a_{1} e_{1}(t)+a_{2}\left(a_{0}+x_{1}(t) \operatorname{sign} x_{1}(t)\right)^{-1} \\
& -a_{2}\left(a_{0}+x(t) \operatorname{sign} x(t)\right)^{-1}+a_{3} e_{2}(t) \\
& +u_{2},
\end{aligned}
$$

where $e_{1}=x_{1}-x$ and $e_{2}=y_{1}-y$.

Theorem 11. The drive system (11) and the response system (31) are fixed-time synchronization under the following controller:

$$
\begin{aligned}
u_{1}= & -p_{1} e_{1}(t)-\mu \operatorname{sign}\left(e_{1}(t)\right)\left|e_{1}(t)\right|^{\lambda-m / n} \\
& -v \operatorname{sign}\left(e_{1}(t)\right)\left|e_{1}(t)\right|^{m / n}, \\
u_{2}= & -p_{2} e_{2}(t)-\mu \operatorname{sign}\left(e_{2}(t)\right)\left|e_{2}(t)\right|^{\lambda-m / n} \\
& -v \operatorname{sign}\left(e_{2}(t)\right)\left|e_{2}(t)\right|^{m / n} \\
& -a_{2}\left(a_{0}+x_{1}(t) \operatorname{sign} x_{1}(t)\right)^{-1} \\
& +a_{2}\left(a_{0}+x(t) \operatorname{sign} x(t)\right)^{-1},
\end{aligned}
$$

where $m / n<(1+\lambda) / 2<(2 n+m) / 2 n, p_{1}>\left(1-a_{1}\right) / 2$, $p_{2}>a_{3}+\left(1-a_{1}\right) / 2, \mu>v>0, m<n$, and $m+n$ and $2 n$ are coprime positive integers.

Proof. Let $V(t)=(1 / 2) e_{1}^{2}(t)+(1 / 2) e_{2}^{2}(t)$, and so

$$
\begin{aligned}
& \dot{V}(t)=e_{1}(t) \dot{e}_{1}(t)+e_{2}(t) \dot{e}_{2}(t)=e_{1}(t)\left(e_{2}(t)\right. \\
& -p_{1} e_{1}(t)-\mu \operatorname{sign}\left(e_{1}(t)\right)\left|e_{1}(t)\right|^{\lambda-m / n} \\
& \left.\quad-v \operatorname{sign}\left(e_{1}(t)\right)\left|e_{1}(t)\right|^{m / n}\right)+e_{2}(t)\left(-a_{1} e_{1}(t)\right. \\
& \quad+a_{3} e_{2}(t)-p_{2} e_{2}(t)-\mu \operatorname{sign}\left(e_{2}(t)\right)\left|e_{2}(t)\right|^{\lambda-m / n}
\end{aligned}
$$

$$
\begin{aligned}
& \left.-v \operatorname{sign}\left(e_{2}(t)\right)\left|e_{2}(t)\right|^{m / n}\right)=\left(1-a_{1}\right) e_{1}(t) e_{2}(t) \\
& -p_{1} e_{1}^{2}(t)-\left(p_{2}-a_{3}\right) e_{2}^{2}(t) \\
& -\mu\left(e_{1}(t) \operatorname{sign}\left(e_{1}(t)\right)\left|e_{1}(t)\right|^{\lambda-m / n}\right. \\
& \left.+e_{2}(t) \operatorname{sign}\left(e_{2}(t)\right)\left|e_{2}(t)\right|^{\lambda-m / n}\right) \\
& -v\left(e_{1}(t) \operatorname{sign}\left(e_{1}(t)\right)\left|e_{1}(t)\right|^{m / n}\right. \\
& \left.+e_{2}(t) \operatorname{sign}\left(e_{2}(t)\right)\left|e_{2}(t)\right|^{m / n}\right) \leq \frac{\left(1-a_{1}\right)}{2}\left(e_{1}^{2}(t)\right. \\
& \left.+e_{2}^{2}(t)\right)-p_{1} e_{1}^{2}(t)-\left(p_{2}-a_{3}\right) e_{2}^{2}(t) \\
& -\mu\left(e_{1}(t) \operatorname{sign}\left(e_{1}(t)\right)\left|e_{1}(t)\right|^{\delta-m / n}\right. \\
& \left.+e_{2}(t) \operatorname{sign}\left(e_{2}(t)\right)\left|e_{2}(t)\right|^{\lambda-m / n}\right) \\
& -v\left(e_{1}(t) \operatorname{sign}\left(e_{1}(t)\right)\left|e_{1}(t)\right|^{m / n}\right. \\
& \left.+e_{2}(t) \operatorname{sign}\left(e_{2}(t)\right)\left|e_{2}(t)\right|^{m / n}\right)=-\left(p_{1}-\frac{1-a_{1}}{2}\right) \\
& \cdot e_{1}^{2}(t)-\left(p_{2}-a_{3}-\frac{1-a_{1}}{2}\right) e_{2}^{2}(t) \\
& -\mu\left(e_{1}(t) \operatorname{sign}\left(e_{1}(t)\right)\left|e_{1}(t)\right|^{\lambda-m / n}\right. \\
& \left.+e_{2}(t) \operatorname{sign}\left(e_{2}(t)\right)\left|e_{2}(t)\right|^{\lambda-m / n}\right) \\
& -v\left(e_{1}(t) \operatorname{sign}\left(e_{1}(t)\right)\left|e_{1}(t)\right|^{m / n}\right. \\
& \left.+e_{2}(t) \operatorname{sign}\left(e_{2}(t)\right)\left|e_{2}(t)\right|^{m / n}\right) \\
& \leq-\mu\left(\left|e_{1}(t)\right|^{1+\lambda-m / n}+\left|e_{2}(t)\right|^{1+\lambda-m / n}\right) \\
& -v\left(\left|e_{1}(t)\right|^{1+m / n}+\left|e_{2}(t)\right|^{1+m / n}\right) \leq-\mu\left(\left|e_{1}(t)\right|^{2}\right. \\
& \left.+\left|e_{2}(t)\right|^{2}\right)^{(1+\lambda) / 2-m / 2 n}-v\left(\left|e_{1}(t)\right|^{2}\right. \\
& \left.+\left|e_{2}(t)\right|^{2}\right)^{1 / 2+m / 2 n}=-\mu 2^{(1+\lambda) / 2-m / 2 n}\left(\frac{1}{2}\left|e_{1}(t)\right|^{2}\right. \\
& \left.+\frac{1}{2}\left|e_{2}(t)\right|^{2}\right)^{(1+\lambda) / 2-m / 2 n}-v 2^{1 / 2+m / 2 n}\left(\frac{1}{2}\left|e_{1}(t)\right|^{2}\right. \\
& \left.+\frac{1}{2}\left|e_{2}(t)\right|^{2}\right)^{1 / 2+m / 2 n} \\
& =-\mu 2^{(1+\lambda) / 2-m / 2 n}(V(t))^{(1+\lambda) / 2-m / 2 n} \\
& -v 2^{1 / 2+m / 2 n}(V(t))^{1 / 2+m / 2 n} .
\end{aligned}
$$




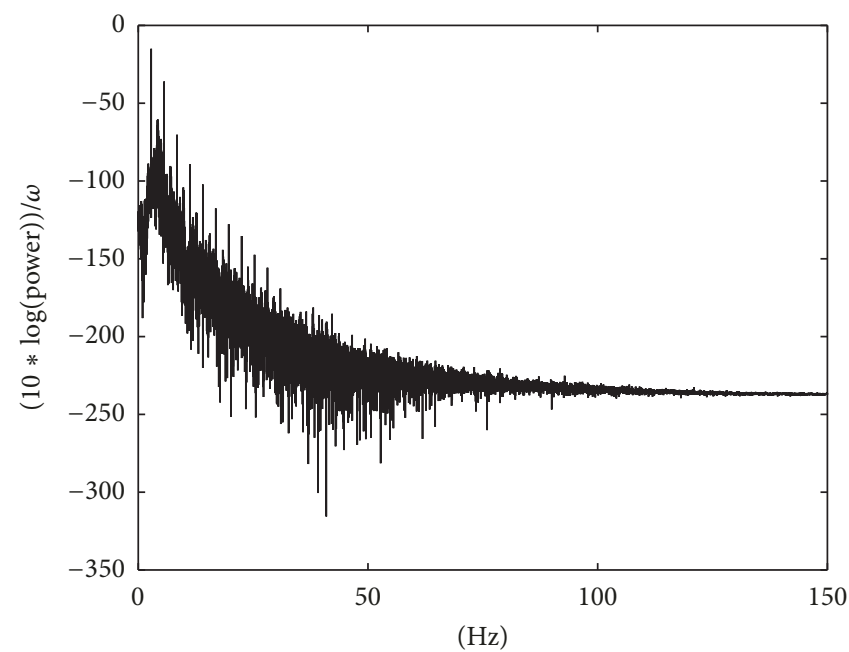

FIgURE 5: Spectrum diagram.

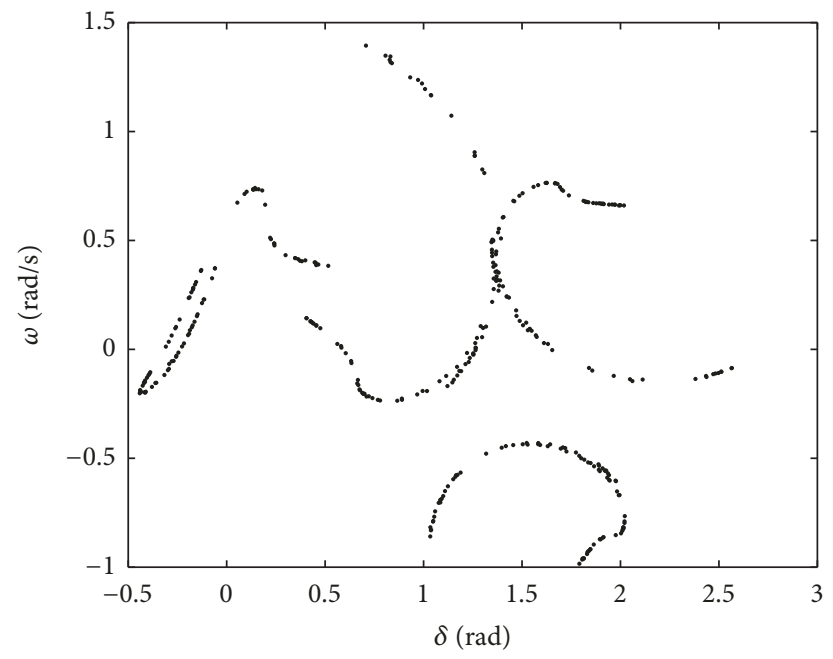

Figure 6: Poincare diagram.

By Lemma 6 and Comparison Principle of differential equations [28], we obtain

$$
\lim _{t \rightarrow T} V(t)=0,
$$

where the settling time is given by

$$
\begin{aligned}
T< & \frac{1}{\mu 2^{(1+\lambda) / 2-m / 2 n}} \frac{1}{(1+\lambda) / 2-(2 n+m) / 2 n} \\
& +\frac{1}{\nu 2^{1 / 2+m / 2 n}} \frac{2 n}{(2 n-m)} .
\end{aligned}
$$

In numerical simulation, let $a_{0}=0.15, a_{1}=0.6, a_{2}=0.5$, $a_{3}=-0.2, w=0.6, q=0.8, \lambda=2, m=1, n=2, p_{1}=1, p_{2}=$ $1, \mu=2$, and $\nu=1$. By calculation from (36), the control time is obtained: $T<1.6337$. The initial values of the state are $2,-2$, 6, and 3; the error evolution is shown in Figure 7. Numerical simulations illustrate the effectiveness of Theorem 11.
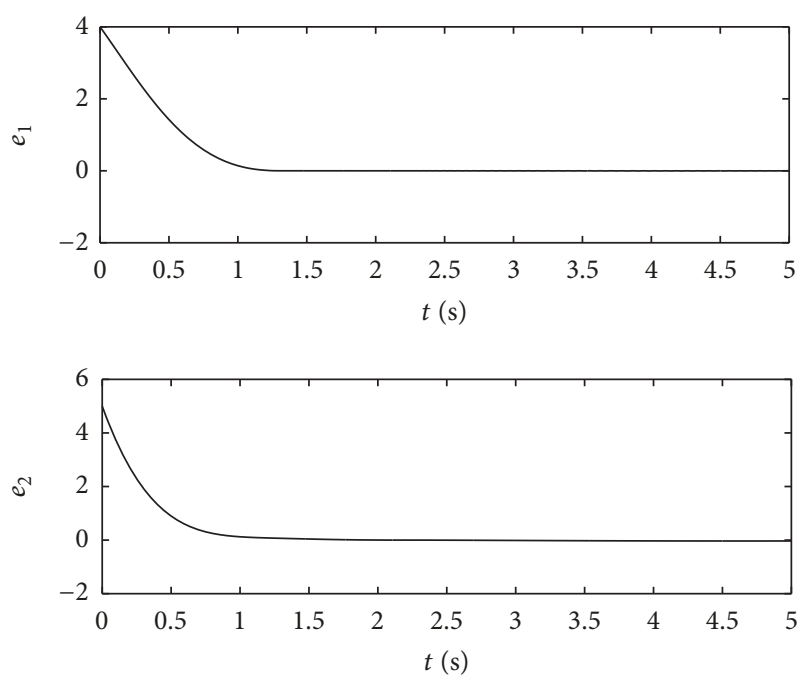

FIGURE 7: Synchronization errors. 


\section{Conclusion}

Chaos and bifurcation are complex phenomena in nonlinear economic and financial systems. In this paper, the stock price fluctuation dynamic system has been established according to the rule of the stock market, and basic dynamic properties of the system has been analyzed by external excitations, such as the equilibrium point and stability, Lyapunov exponent, fractal dimension, and bifurcation diagram. At the same time, the new fixed-time convergence theorem has been proposed in this paper, and the fixed-time control criterion of the stock price fluctuation system has also been studied. Our future work is to study dynamic properties of stock price fluctuation system with time-delay.

\section{Conflicts of Interest}

The authors declare that there are no conflicts of interest regarding the publication of this paper.

\section{Acknowledgments}

This work is supported by the National Natural Science Foundation of China (61673221, 61673257, and 11701287), the Youth Fund Project of the Humanities and Social Science Research for the Ministry of Education of China (14YJCZH173), Top-Notch Academic Programs Project of Jiangsu Higher Education Institutions (Jiangsu Province Office, no. [2015] 1, PPZY2015B104), the Key Laboratory of Financial Engineering of Jiangsu Province (NSK2015-16), Applied Economics of key Sequence Disciplines of Jiangsu Higher Education Institutions (Jiangsu Province Office, no. [2014] 37), "Qing-Lan Engineering” Foundation of Jiangsu Higher Education Institutions, and a project funded by the Priority Academic Program Development of Jiangsu Higher Education Institutions (PAPD).

\section{References}

[1] E. N. Lorenz, "Deterministic nonperiodic flow," Journal of the Atmospheric Sciences, vol. 20, no. 2, pp. 130-141, 1963.

[2] P. Chen, "Empirical and theoretical evidence of economic chaos," System Dynamics Review, vol. 4, no. 1-2, pp. 81-108, 1988.

[3] C. Xie, Y. Xu, and D. Tong, "Chaos synchronization of financial chaotic system with external perturbation," Discrete Dynamics in Nature and Society, vol. 2015, Article ID 731376, 7 pages, 2015.

[4] J. Ma, H. Ren, M. Yu, and M. Zhu, "Research on the complexity and chaos control about a closed-loop supply chain with dual-channel recycling and uncertain consumer perception," Complexity, vol. 2018, Article ID 9853635, 13 pages, 2018.

[5] Y. Li, S. Zhang, and J. Han, "Dynamic pricing and periodic ordering for a stochastic inventory system with deteriorating items," Automatica, vol. 76, pp. 200-213, 2017.

[6] F. Grassetti and G. Hunanyan, "On the economic growth theory with Kadiyala production function," Communications in Nonlinear Science and Numerical Simulation, 2017.

[7] R. Arévalo, J. García, F. Guijarro, and A. Peris, "A dynamic trading rule based on filtered flag pattern recognition for stock market price forecasting," Expert Systems with Applications, vol. 81, pp. 177-192, 2017.
[8] J. Zhang, L. Lei, S. Zhang, and L. Song, "Dynamic vs. static pricing in a supply chain with advertising," Computers \& Industrial Engineering, vol. 109, pp. 266-279, 2017.

[9] J. A. Primbs and B. R. Barmish, "On robustness of simultaneous long-short stock trading control with time-varying price dynamics," IFAC-PapersOnLine, vol. 50, no. 1, pp. 12267-12272, 2017.

[10] M. Cai and H. Cao, "Bifurcations of periodic orbits in Duffing equation with periodic damping and external excitations," Nonlinear Dynamics, vol. 70, no. 1, pp. 453-462, 2012.

[11] E. E. Mahmoud and F. S. Abood, "A new nonlinear chaotic complex model and its complex antilag synchronization," Complexity, vol. 2017, Article ID 3848953, 13 pages, 2017.

[12] X.-T. Tran and H.-J. Kang, "Fixed-time complex modified function projective lag synchronization of chaotic (hyperchaotic) complex systems," Complexity, vol. 2017, Article ID 4020548, 9 pages, 2017.

[13] Y.-W. Wang, W. Yang, J.-W. Xiao, and Z.-G. Zeng, "Impulsive multisynchronization of coupled multistable neural networks with time-varying delay," IEEE Transactions on Neural Networks and Learning Systems, vol. 28, no. 7, pp. 1560-1571, 2017.

[14] Y. Tang, H. Gao, W. Zou, and J. Kurths, "Distributed synchronization in networks of agent systems with nonlinearities and random switchings," IEEE Transactions on Systems, Man, and Cybernetics, Part B: Cybernetics, vol. 43, no. 1, pp. 358-370, 2013.

[15] W. Gao, Y. Li, and Y. Xu, "Parameters estimation and adaptive synchronization of chaotic systems with adaptive parameters perturbation," in Proceedings of the 2012 International Conference on Industrial Control and Electronics Engineering, ICICEE 2012, pp. 746-749, China, August 2012.

[16] J. Lu, J. Kurths, J. Cao, N. Mahdavi, and C. Huang, "Synchronization control for nonlinear stochastic dynamical networks: Pinning impulsive strategy," IEEE Transactions on Neural Networks and Learning Systems, vol. 23, no. 2, pp. 285-292, 2012.

[17] H. Shen, J. H. Park, and Z.-G. Wu, "Finite-time synchronization control for uncertain Markov jump neural networks with input constraints," Nonlinear Dynamics, vol. 77, no. 4, pp. 1709-1720, 2014.

[18] A. Polyakov, "Nonlinear feedback design for fixed-time stabilization of linear control systems," Institute of Electrical and Electronics Engineers Transactions on Automatic Control, vol. 57, no. 8, pp. 2106-2110, 2012.

[19] J. D. Cao and R. X. Li, "Fixed-time synchronization of delayed memristor-based recurrent neural networks," Science China Information Sciences, vol. 60, no. 3, Article ID 032201, 2017.

[20] A. Heydari and S. N. Balakrishnan, "Fixed-final-time optimal tracking control of input-affine nonlinear systems," Neurocomputing, vol. 129, pp. 528-539, 2014.

[21] Z. Song and J. Zhai, "Finite-time adaptive control for a class of switched stochastic uncertain nonlinear systems," Journal of The Franklin Institute, vol. 354, no. 12, pp. 4637-4655, 2017.

[22] H. Zhao, L. Li, H. Peng, J. Xiao, Y. Yang, and M. Zheng, "Finitetime topology identification and stochastic synchronization of complex network with multiple time delays," Neurocomputing, vol. 219, pp. 39-49, 2017.

[23] Z. Zuo, "Nonsingular fixed-time consensus tracking for secondorder multi-agent networks," Automatica, vol. 54, pp. 305-309, 2015.

[24] J. Ni, L. Liu, C. Liu, X. Hu, and T. Shen, "Fixed-time dynamic surface high-order sliding mode control for chaotic oscillation in power system," Nonlinear Dynamics, vol. 86, no. 1, pp. 401420, 2016. 
[25] M. Defoort, A. Polyakov, G. Demesure, M. Djemai, and K. Veluvolu, "Leader-follower fixed-time consensus for multiagent systems with unknown non-linear inherent dynamics," IET Control Theory \& Applications, vol. 9, no. 14, pp. 2165-2170, 2015.

[26] J. Fu and J. Wang, "Fixed-time coordinated tracking for secondorder multi-agent systems with bounded input uncertainties," Systems Control Letters, vol. 93, pp. 1-12, 2016.

[27] Y. Shang and Y. Ye, "Leader-follower fixed-time group consensus control of multiagent systems under directed topology," Complexity, vol. 2017, Article ID 3465076, 2017.

[28] T. Yun, "Basic equations, theory and principle of computational stock market," Applied Mathematics and Mechanics, vol. 20, no. 2, pp. 145-151, 1999.

[29] A. K. Tiwari, C. Oros, and C. T. Albulescu, "Revisiting the inflation-output gap relationship for France using a wavelet transform approach," Economic Modelling, vol. 37, pp. 464-475, 2014.

[30] J. Lü, G. Chen, and D. Cheng, "A new chaotic system and beyond: the generalized Lorenz-like system," International Journal of Bifurcation and Chaos, vol. 14, no. 5, pp. 1507-1537, 2004.

[31] G. H. Hardy, J. E. Littlewood, and G. Pólya, Inequalities, Cambridge University Press, London, UK, 2nd edition, 1952. 


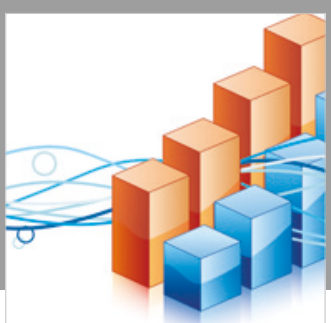

Advances in

Operations Research

\section{-n-m}
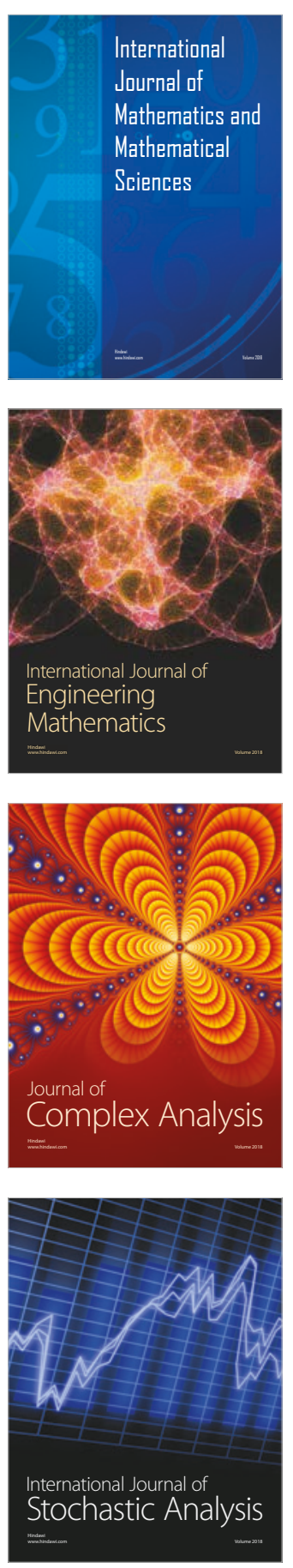
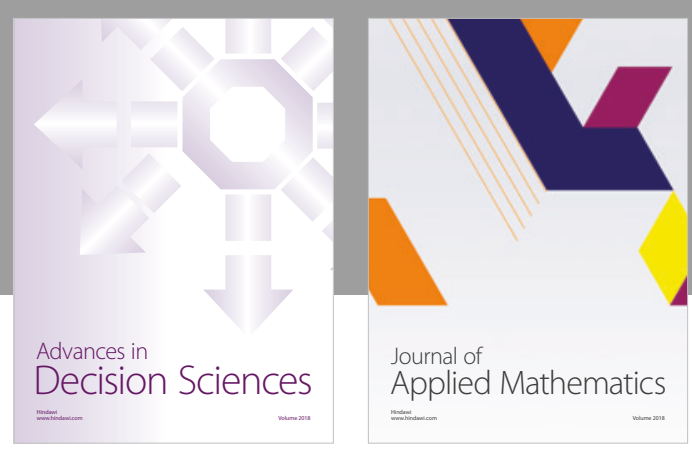

Journal of

Applied Mathematics
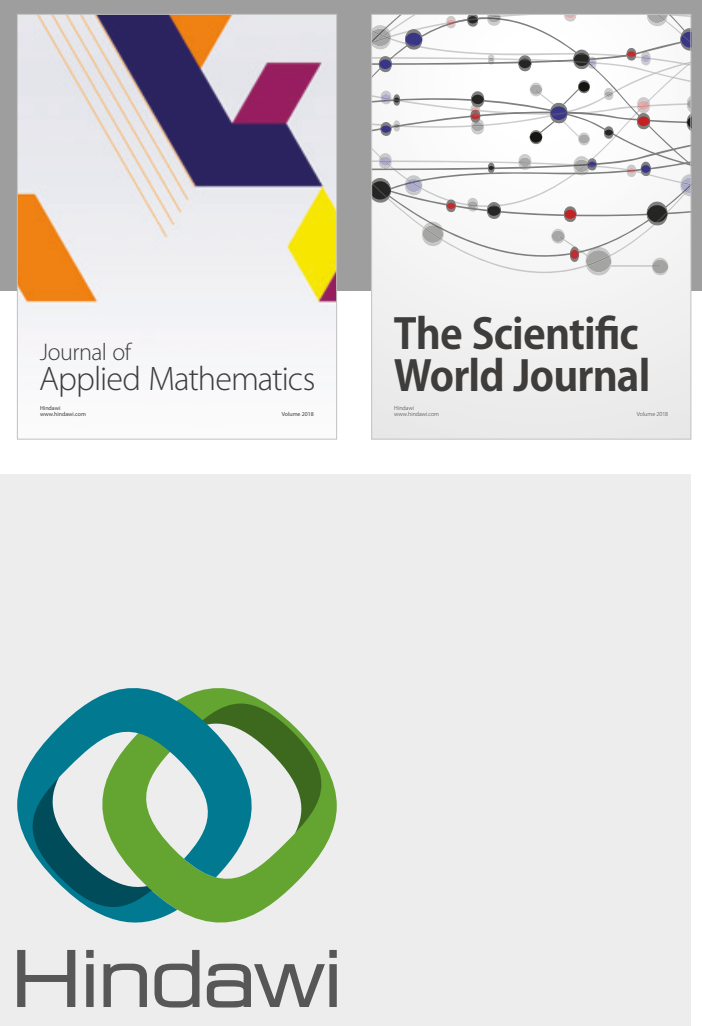

Submit your manuscripts at

www.hindawi.com

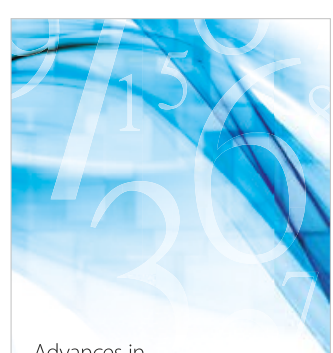

Advances in
Numerical Analysis
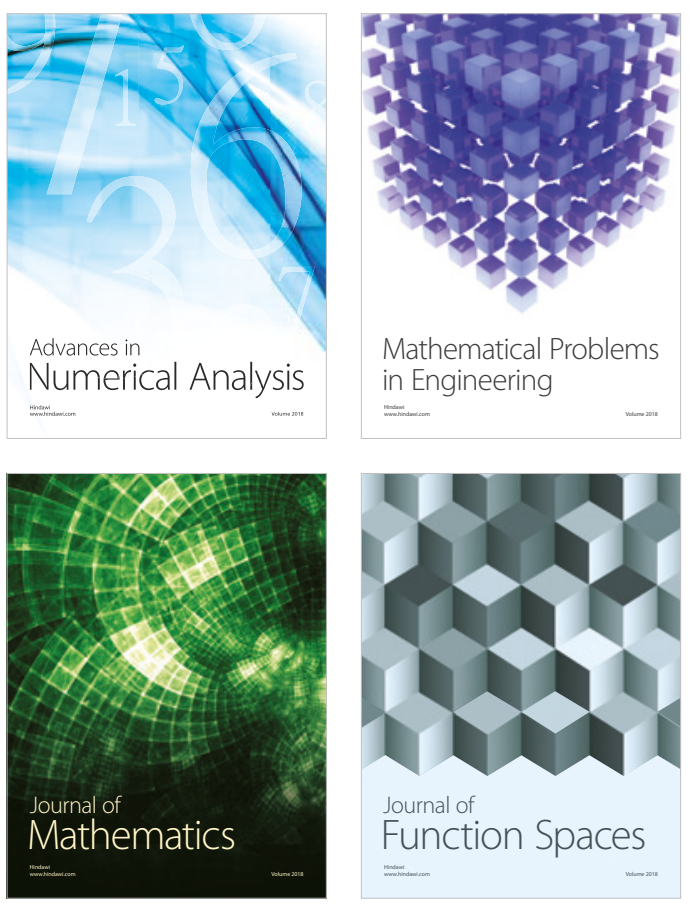

Mathematical Problems in Engineering

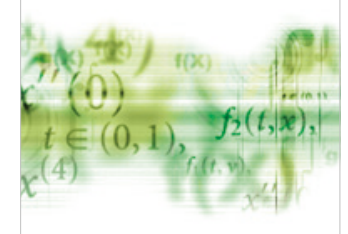

International Journal of

Differential Equations

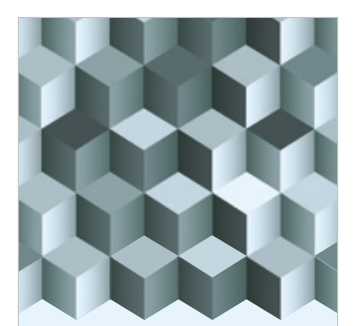

Journal of

Function Spaces
The Scientific

World Journal

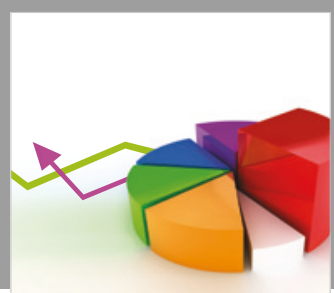

Journal of

Probability and Statistics
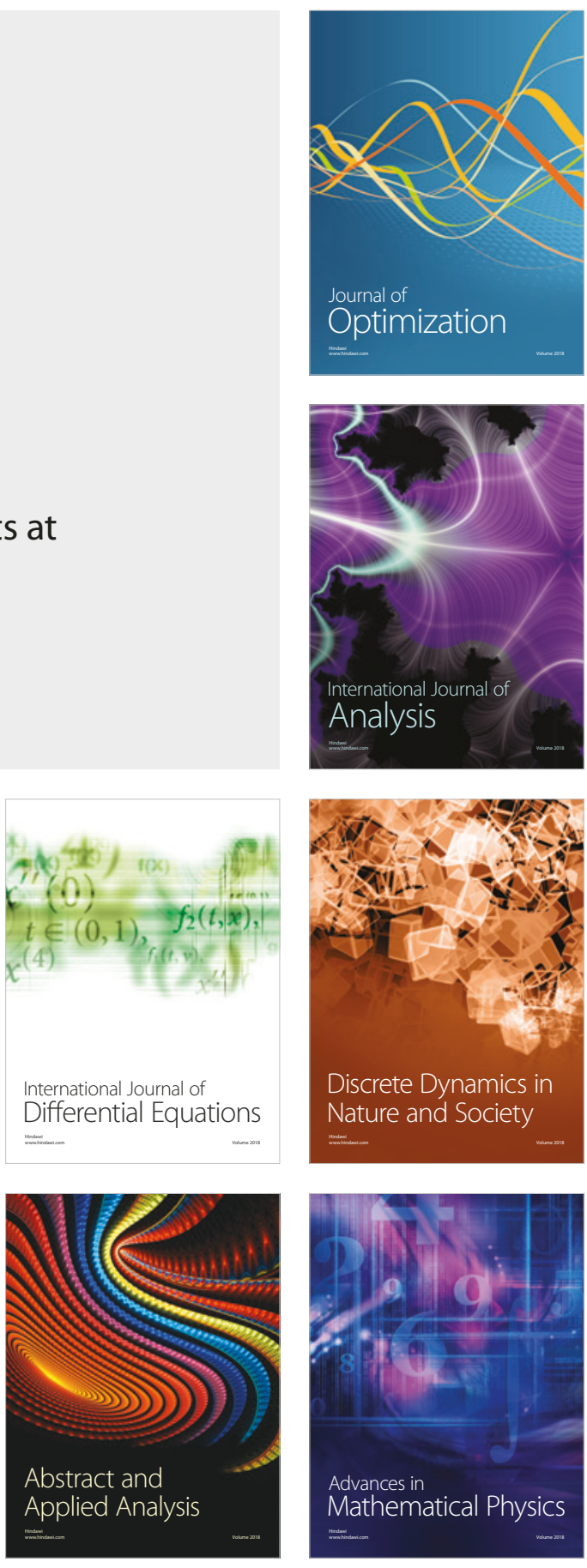